\title{
Convergent vomeronasal system reduction in mammals coincides with convergent losses of calcium signalling and odorant-degrading genes
}

\author{
Nikolai Hecker ${ }^{1,2,3}$ (D) | Ulla Lächele ${ }^{4}$ | Heiko Stuckas ${ }^{5,6}$ (D) | Peter Giere ${ }^{4}$ | \\ Michael Hiller ${ }^{1,2,3}$
}

${ }^{1}$ Center for Systems Biology Dresden, Dresden, Germany

${ }^{2}$ Max Planck Institute of Molecular Cell Biology and Genetics, Dresden, Germany

${ }^{3}$ Max Planck Institute for the Physics of Complex Systems, Dresden, Germany

${ }^{4}$ Museum für Naturkunde, Leibniz Institute for Evolution and Biodiversity Science, Berlin, Germany

${ }^{5}$ Population Genetics, Senckenberg Natural History Collections Dresden, Dresden, Germany

${ }^{6}$ Leibniz Institution for Biodiversity and Earth System Research, Dresden, Germany

\section{Correspondence}

Nikolai Hecker, Computational Biology and Evolutionary Genomics, Center for Systems Biology Dresden, Max Planck Institute of Molecular Cell Biology and Genetics \& Max Planck Institute for the Physics of Complex Systems, Dresden, Germany.

Email: hecker@mpi-cbg.de

\section{Funding information}

Max Planck Society; Leibniz Association, Grant/Award Number: SAW-2016-SGN-2

\begin{abstract}
The vomeronasal system (VNS) serves crucial functions for detecting olfactory clues often related to social and sexual behaviour. Intriguingly, two of the main components of the VNS, the vomeronasal organ (VNO) and the accessory olfactory bulb, are regressed in aquatic mammals, several bats and primates, likely due to adaptations to different ecological niches. To detect genomic changes that are associated with the convergent reduction of the VNS, we performed the first systematic screen for convergently inactivated protein-coding genes associated with convergent VNS reduction, considering 106 mammalian genomes. Extending previous studies, our results support that $\operatorname{Trpc} 2$, a cation channel that is important for calcium signalling in the VNO, is a predictive molecular marker for the presence of a VNS. Our screen also detected the convergent inactivation of the calcium-binding protein $\mathrm{S} 100 \mathrm{z}$, the aldehyde oxidase Aox 2 that is involved in odorant degradation, and the uncharacterized MsInl gene that is expressed in the VNO and olfactory epithelium. Furthermore, we found that Trpc2 and S100z or Aox 2 are also inactivated in otters and Phocid seals for which no morphological data about the VNS are available yet. This predicts a VNS reduction in these semi-aquatic mammals. By examining the genomes of 115 species in total, our study provides a detailed picture of how the convergent reduction of the VNS coincides with gene inactivation in placental mammals. These inactivated genes provide experimental targets for studying the evolution and biological significance of the olfactory system under different environmental conditions.
\end{abstract}

\section{KEYWORDS}

accessory olfactory bulb, calcium signalling, convergent gene loss, odorant degradation,

vomeronasal organ, vomeronasal system

\section{1 | INTRODUCTION}

Recent developments in comparative genomics provide insights into genomic changes that are associated with different environmental

Nikolai Hecker and Ulla Lächele should be considered joint first author. adaptations during mammalian evolution. In this context, the inactivation of protein-coding, also referred to as gene loss, has recently received more and more attention (Albalat \& Canestro, 2016; Sharma et al., 2018). The inactivation or loss of protein-coding genes reflects adaptations to diverse ecological niches including the change to a subterranean or aquatic habitat (Chikina, Robinson, \& Clark, 2016; Ehrlich 
et al., 2019; Huelsmann et al., 2019; Kishida, Kubota, Shirayama, \& Fukami, 2007; Lopes-Marques et al., 2019; Nery, Arroyo, \& Opazo, 2014; Partha et al., 2017; Prudent, Parra, Schwede, Roscito, \& Hiller, 2016; Sharma et al., 2018) and various nutrition strategies that range from carnivory, herbivory, insectivory to frugivory (Hecker, Sharma, \& Hiller, 2019; Huelsmann et al., 2019; Jiang et al., 2012; Kim et al., 2016; Liu et al., 2016; Lopes-Marques et al., 2019; Sharma et al., 2018). Hence, studying the inactivation of protein-coding genes constitutes a promising approach to gain further insights into evolutionary ecology.

In this context, changes in olfaction play important roles for mammalian evolution. Olfaction contributes to essential aspects of mammalian ecology such as the search for food, discrimination between poisonous and nonpoisonous food, navigation, detection of threats like predators and also social and sexual interaction (Stoddart, 1980). As such, olfaction is a crucial sense that drives adaptations to diverse ecological niches and affects the interaction between organisms. In this study, we focus on the evolutionary changes in olfaction that are largely related to sociosexual behaviour and ecological adaption in mammals: the reduction of the vomeronasal system.

Most mammals possess two olfactory systems: the main olfactory system and the vomeronasal system which synergistically mediate the sense of smell (Kelliher, 2007; Suarez, Garcia-Gonzalez, \& Castro, 2012). The main olfactory system detects odorants through the olfactory epithelium and processes their detection in the main olfactory bulb, whereas the vomeronasal system (VNS) forms an accessory olfactory pathway with distinct organs (Figure 1). Two of the main components of the VNS are the vomeronasal organ (VNO) and the accessory olfactory bulb (AOB; D'Aniello, Semin, Scandurra, \& Pinelli, 2017). In the VNO, primary sensory neurons are activated by nonvolatile or ephemeral chemical cues and send signals to the accessory olfactory bulb (Dulac, 2000; Stowers \& Marton, 2005). Although there are clear indications that the main olfactory system and VNS are functionally intertwined (Kelliher, 2007; Stowers \& Marton, 2005;

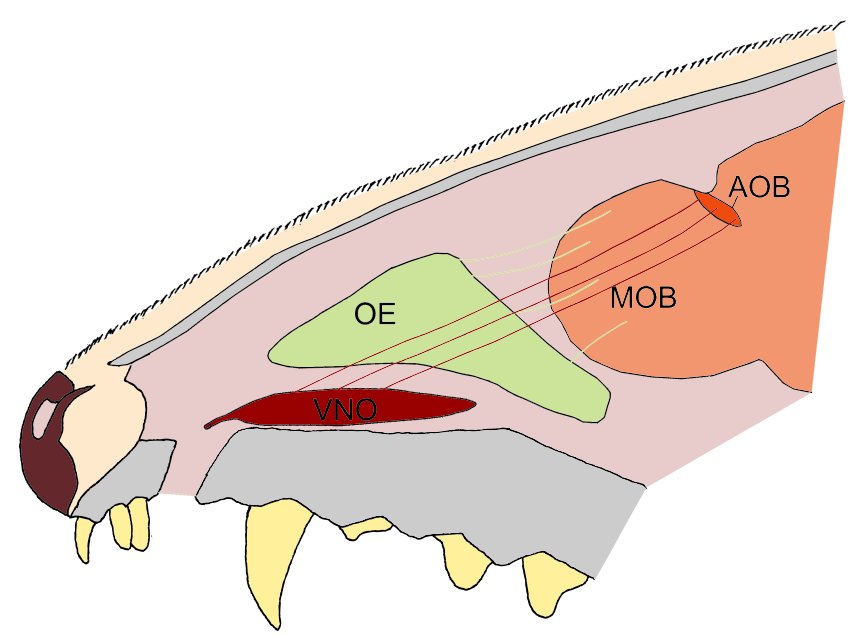

FIGURE 1 Schematic lateral representation of relevant components of the olfactory system in a hypothetical mammal. $\mathrm{AOB}$, accessory olfactory bulb; $\mathrm{MOB}$, main olfactory bulb; $\mathrm{OE}$, olfactory epithelium; VNO, vomeronasal organ [Colour figure can be viewed at wileyonlinelibrary.com]
Suarez et al., 2012), activity patterns in the neural vomeronasal pathway, ablation of the VNO and arguably knockout studies suggest that the mammalian VNS is of special importance for sensing chemical cues that affect social and sexual behaviours such as the detection of mating partners, parental care and territorial aggression (Bean \& Wysocki, 1989; Stowers, Holy, Meister, Dulac, \& Koentges, 2002; Tachikawa, Yoshihara, \& Kuroda, 2013; Yu, 2015).

Despite the importance of the VNS for sociosexual behaviour, several mammalian species show a regression of the $\mathrm{VNO}$ during development. In 1813, Ludvig Jacobson assumed that a VNO is present in all mammals (Doving \& Trotier, 1998). Studies about reptiles and amphibians suggest that the VNO was an innovation of tetrapods (Burton, 1990; Burton, Coogan, \& Borror, 1990; Wang \& Halpern, 1980). However, also lungfish appear to possess a VNO (Bertmar, 1969; Gonzalez, Morona, Lopez, Moreno, \& Northcutt, 2010; Wittmer \& Nowack, 2017). While, for instance, teleost fish do not possess a VNO, the presence of VNO-related genes in diverse vertebrate species suggests that VNS function evolved in the common ancestor of vertebrates (Grus \& Zhang, 2009; Pfister \& Rodriguez, 2005; Zapilko \& Korsching, 2016). However, more elaborate studies on mammals found that several species exhibit a reduced VNO or lack a VNO altogether (Bhatnagar \& Meisami, 1998). The VNO was lost secondarily in multiple bat species, cetaceans, the manatee and in higher primates (Bhatnagar \& Meisami, 1998; Mackay-Sim, Duvall, \& Graves, 1985; Oelschläger, Buhl, \& Dann, 1987). Consistent with the assumption that a functional VNS requires both a VNO and AOB, reduction or loss of the $\mathrm{VNO}$ is often associated with a reduction or loss of the AOB (Meisami \& Bhatnagar, 1998). The presence or absence of the VNS shows at least partly an environmental dependency as indicated by its independent secondary loss in aquatic mammals, bats and primates (Figure 2; Bhatnagar \& Meisami, 1998; Mackay-Sim et al., 1985; Oelschläger et al., 1987). This may suggest that environmental and niche-specific adaptations have resulted in relaxed constraint on the VNS, which ultimately lead to its reduction or loss.

The evolutionary reduction of the VNS is reflected by the convergent inactivation and contraction of VNO-related protein-coding genes. For example, the number of vomeronasal receptor genes is highly contracted in cetaceans, bats, apes and Old World monkeys (Young, Massa, Hsu, \& Trask, 2010). Furthermore, the inactivation of the VNO-specific cation channel Trpc2 has previously been described in cetaceans, several bat species and catarrhines which all lack a functional VNO (Liman \& Innan, 2003; Yohe et al., 2017; Yu et al., 2010). However, these studies focused on selected candidate genes and investigated either only a small set of species or a specific clade. To overcome these limitations and to gain a broader understanding into genomic changes associated with VNS reduction, we performed the first systematic screen for convergently inactivated protein-coding genes associated with the convergent reduction of the VNS using the forward genomics principle (Hecker et al., 2019; Hiller et al., 2012; Sharma et al., 2018). Considering 106 placental mammals, we detected a clear association between the inactivation of Trcp2 and the reduction of the VNS. Our screen also discovered the inactivation of 


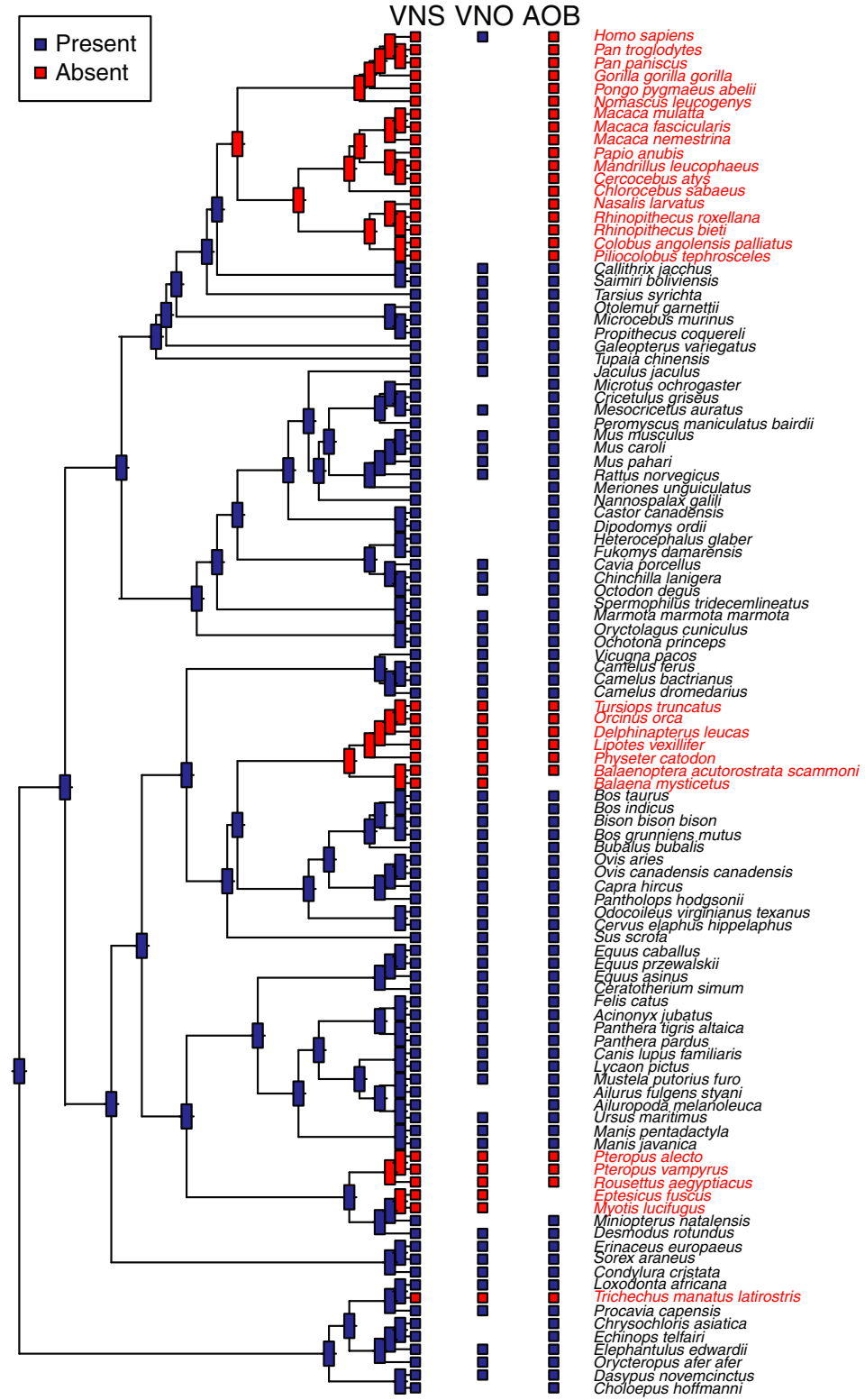

FIGURE 2 Convergent reduction of the vomeronasal system in mammals. The presence or absence of the vomeronasal organ (VNO) and accessory olfactory bulb (AOB) in adult placental mammals is indicated by blue and red rectangles, respectively. No rectangle indicates that no data were available about the VNO or $A O B$. The VNS was defined as absent if either the $\mathrm{VNO}$ or $\mathrm{AOB}$ was absent and otherwise treated as present. Internal nodes of the phylogenetic tree visualize the likelihood that the VNS was present (blue) or absent (red) in the respective ancestors. Ancestral phenotype reconstruction indicates that all ancestral states could be assigned as either "VNS present" or "VNS absent" with a high likelihood. Families in which the VNS was lost are indicated on the right side. The regression of the $\mathrm{VNO}$ and $\mathrm{AOB}$ co-occurs in all mammals for which morphological data about the presence or absence of both organs were available, except for humans. Although there is an ongoing debate about the regression of the VNO in adult humans, the lack of sensory neurons suggests that the human VNO is not functional (D'Aniello et al., 2017; Meredith, 2001) [Colour figure can be viewed at wileyonlinelibrary.com]

\section{Chiroptera}

Sirenia additional genes with roles in calcium signalling and odorant degradation. By extending our analysis to nine additional genomes, we found the inactivation of these genes in Phocid seals and otters for which no morphological data about the VNS exist. This suggests a reduction of the VNS in these semi-aquatic species and provides novel insights into habitat- and niche-dependent genomic changes during evolution.

\section{2 | MATERIAL AND METHODS}

\subsection{Analysis of the evolutionary reduction of the vomeronasal system}

As prerequisite for identifying genomic changes associated with the reduction of the vomeronasal system (VNS) in placental mammals, morphological data about the presence or absence of the $\mathrm{VNO}$ and $\mathrm{AOB}$ were compiled based on a comprehensive literature research and by inspection of zoological collections. Here, we considered embryonic as well as adult stages of the species. We defined the VNS to be absent if the VNO or the AOB was absent in adults. After mapping the presence and absence of the VNS to the phylogeny of placental mammals, we reconstructed ancestral states of VNS presence/absence with the R function "ace" from the "ape" package using a maximum-likelihood estimation ( method = "ML"), discrete character encodings and equal rates (type = "ER"; Felsenstein, 1973; Schluter, Price, Mooers, \& Ludwig, 1997).

\subsection{Identification of gene inactivating mutations}

In order to identify inactivated protein-coding genes, a previously published approach for identifying gene inactivating mutations was employed (Sharma et al., 2018). This approach detects frameshift mutations, splice-site mutations, premature stop codons and exon deletions based on whole-genome alignments and reference gene structures and is able to distinguish between genome assembly 
gaps and real deletions. As input, we used a whole-genome alignment between the mouse genome assembly mm10 and genome assemblies of 115 mammalian species (Table S1) obtained with lastz, axtChain, chainCleaner and Multiz-TBA with lastz parameters $K=2,400, L=3,000$ and default parameters for other methods (Blanchette et al., 2004; Harris, 2007; Kent, Baertsch, Hinrichs, Miller, \& Haussler, 2003; Suarez, Langer, Ladde, \& Hiller, 2017). For each species, we only kept parts of the alignment which consisted of long chains of aligned blocks (score $\geq 100,000$ ). To infer the correct reading frame, exon sequences were realigned with CESAR based on all principal isoforms of the genes (Mouse GRCm38 GencodeM16/Ensembl91) obtained from APPRIS (Rodriguez et al., 2018; Sharma, Elghafari, \& Hiller, 2016; Sharma, Schwede, \& Hiller, 2017). We excluded olfactory receptors from this analysis and analysed all other 19,710 protein-coding genes. A gene was classified as inactivated if the maximum percentage of the reading frame that remains intact (\%intact) was $<80 \%$. This \%intact value is computed based on the positions of inactivating mutations (Sharma et al., 2018). For sequences which lacked a start codon AUG, we searched the first in-frame AUG triplet and adjusted the \%intact. If a gene has only inactivating mutations close to the $\mathrm{N}$ or $\mathrm{C}$ terminus, it might still be functional and will have a high \%intact value. Hence, we considered a gene to be intact if \%intact $\geq 90 \%$. To visualize the overlap between inactivating mutations and known protein domains, we downloaded the available Pfam domain annotation from the UCSC GenomeBrowser (El-Gebali et al., 2019; Haeussler et al., 2019).

\section{3 | Screen for convergently inactivated protein- coding genes}

We used a forward genomics approach (Hecker et al., 2019; Hiller et al., 2012; Sharma et al., 2018) to identify genes that were preferentially inactivated in mammals with a reduced VNS, but that are intact in most mammals with a morphologically intact VNS. We grouped species into the five independent lineages that lost an essential component (VNO or AOB or both) of the VNS. If at least $33 \%$ of the species of a lineage contained an inactivated version of the gene (\%intact $<80 \%$, considering only species present in the alignment), we considered the gene to be inactivated in the lineage. We limited this analysis to protein-coding genes for which we could align at least $50 \%$ of the species with a reduced VNS and $50 \%$ of species with intact VNS. We then selected genes that were inactivated in at least 3 lineages and in $>50 \%$ of the species without complete VNS, but were lost in less than $10 \%$ and intact (\%intact $>90 \%$ ) in at least $80 \%$ of the species with intact VNS.

\section{4 | Validation of inactivating mutations}

To ensure that the inactivated genes were genuine, we manually inspected pairwise genome alignments for synteny with regard to upstream and downstream genes, the absence of assembly gaps, and that sequences were unambiguously aligned to the correct ortholog using the UCSC genome browser and removed sequences which did not satisfy these criteria (Haeussler et al., 2019). Next, we validated premature stop codons, frameshifting insertions and deletions, and splice-site mutations in the remaining sequences by the presence of the same mutations in sister species and unassembled DNA reads from the NCBI TRACE or Sequence Read Archive (Hecker, Sharma, \& Hiller, 2017; Hecker et al., 2019; Kodama, Shumway, \& Leinonen, 2012).

\subsection{Selection rate analysis}

Given that inactivated protein-coding genes should evolve under no or relaxed selection pressure, we tested the sequences of the convergently inactivated genes for relaxed selection rates with RELAX which is part of the HYPHY framework (version 2.3.14; Pond, Frost, \& Muse, 2005; Wertheim, Murrell, Smith, Kosakovsky Pond, \& Scheffler, 2015). Here, we labelled branches corresponding to species with inactivated genes and their common ancestors as foreground (Figure S14-S22). Prior to examining selection rates, we realigned the input sequences with the phylogeny-aware tool PRANK in codon mode using a fixed phylogenetic tree (parameter -once) and fixing gaps based on phylogenetic inference (parameter -F; Loytynoja \& Goldman, 2008). We consider sequences to be significantly relaxed if RELAX estimated a relaxation of selection rates $(K<1)$ with a $p$-value $<.05$. In addition, we tested for relaxed selection rates in specific phylogenetic subtrees (Appendix S1: Figure S14-S23). This includes sequences which only contained splice-site mutations, a single inactivating mutation near the $\mathrm{C}$ terminus, or sequences of some species which did not contain inactivating mutations, but do not possess a functional VNS (Table S4).

\section{6 | RNA expression data}

Averaged RNAseq data from mouse olfactory epithelium and VNO were downloaded from the ArrayExpress (E-ERAD-169, https://www.ebi.ac.uk/arrayexpress/experiments/E-ERAD-169/; Kolesnikov et al., 2015) and RNAseq data from mouse brain, colon, heart, kidney, liver, lung, muscle, spleen and testis from the ArrayExpress (E-MTAB-2801; Merkin, Russell, Chen, \& Burge, 2012).

\section{3 | RESULTS}

\subsection{Comprehensive identification of evolutionary reductions of the vomeronasal system}

As a prerequisite for identifying genomic changes associated with the reduction of the VNS, we performed comprehensive literature search and inspected data from zoological collections to determine which mammalian species lack essential components of the VNS and which ones possess a morphologically intact VNS (Table S1). A functional VNS requires both the presence of a VNO to detect chemical cues and an $A O B$ to process the corresponding signals (Figure 1). All mammals that we examined developed both a VNO and $\mathrm{AOB}$ 
during embryonic development with the exception of fully aquatic species, that is cetaceans and manatees (Trichechus manatus; Table S1). However, the VNO and AOB are regressed in adult stages in these and other mammals (Table S1, Figure 2). Therefore, we defined the VNS as absent if a species does not exhibit a VNO or an AOB in its adult stage. Combining all available data, we found that essential parts of the VNS are absent in 31 mammalian species (Figure 2). The other 75 mammalian species did not exhibit a loss of the VNO or $\mathrm{AOB}$ as adults indicating a functional VNS in these 75 species. Together, we obtained phenotypic data for 106 mammals that also have sequenced genomes.

Given that our genomic screen ranks candidate gene losses by the number of independent lineages with a reduced VNS in which the gene was lost, we first estimated in which ancestral branches the VNS was convergently lost or reduced during the evolution of placental mammals. For this purpose, we mapped the presence and absence of the VNS to the phylogeny of these 106 species and performed ancestral state reconstruction. We identified five independent lineages in which the VNS was lost (Figure 2). The VNS was lost in the entire catarrhine and cetacean lineages. We estimated that a functional VNS was lost at least twice independently in Chiroptera, once in the common ancestor of Pteropodidae and independently in the common ancestor of Vespertilionidae, though this number maybe higher based on recently published studies that investigated many additional bat species (Yohe et al., 2017; Yohe \& Davalos, 2018). The fifth independent loss of the VNS occurred in the fully aquatic Sirenia lineage, which is represented in our analysis by the manatee (Trichechus manatus).

\subsection{Convergent gene inactivation associated with the reduction of the vomeronasal system}

To uncover genomic changes that could be associated with the loss of the VNS, we screened the genomes of 106 placental mammalian species for protein-coding genes that were preferentially inactivated in independent lineages that lost the VNS. To detect convergent gene losses, we used a slightly improved version of a previously established forward genomics approach (Hecker et al., 2019; Sharma et al., 2018). We required that a gene is lost in at least three out of the five VNS-reduced lineages, was lost in at least $50 \%$ of the species with a reduced VNS, but not lost in more than $10 \%$ of the species with an intact VNS (see Section 2.2 for further details). We excluded olfactory receptor genes whose loss has been previously studied in detail (Niimura \& Nei, 2007). Considering a total of 19,710 genes, our screen resulted in a list of seven VNS-associated candidate gene losses that satisfied our filtering criteria: Trpc2, S100z, Ubqln5, Tmprss11g, MsInl, Aox2 and Kcnmb3 ranked by the number of VNS-reduced lineages which lost the gene and fraction of mammals with reduced VNS that lost the gene (Table S2).

To assess whether the candidate gene losses might be associated with a VNS reduction, we investigated available gene expression data of mouse (Mus musculus) VNO, olfactory epithelium, brain, colon, heart, kidney, liver, lung, muscle, spleen and testis tissues (Figure 3 and Figures S1 and S2). Compared with other genes of the same family, $\mathrm{S100z}$ is highly expressed in VNO and Aox2 is highly expressed in olfactory epithelium (Figure 3). MsInl shows a moderate expression in olfactory epithelium (Figure 3). Tmprss11g and Kcnmb3 both show lower expression levels in VNO and olfactory epithelium when compared to genes of the same family (Figure S1). Ubqln5 is very lowly expressed in VNO or olfactory epithelium (Figure S1) but shows an olfactory epithelium-specific expression during mouse embryonic development (E15) based on in situ hybridization data (GDX: MGI:1918230; Diez-Roux et al., 2011; Smith et al., 2019). Among the candidate genes, Ubqln5 exhibits the highest expression in testis (Figure S2). Except for Ubqln5, the candidate genes show no substantial expression in brain, colon, heart, kidney, liver, lung, muscle, spleen or testis (Figure S2).

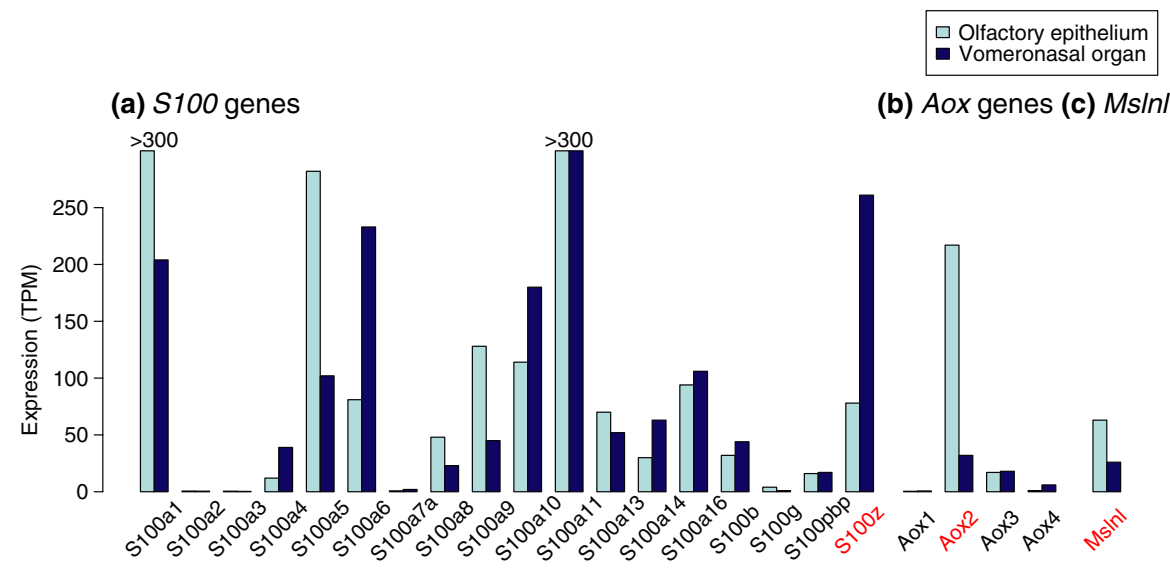

FIGURE 3 RNA expression of convergently inactivated genes in mouse (Mus musculus) olfactory epithelium and vomeronasal organ. The average expression of genes that were convergently inactivated in mammals with reduced VNS (red font) is shown as a bar chart based on available expression data from mouse olfactory epithelium and VNO. For comparison, the expression of members of the same gene families is also shown. $\mathrm{S} 100 \mathrm{z}$ is preferentially expressed in the VNO and is second highest expressed S100 gene in this organ. Aox2 and MsInl show a clear expression in both VNO and olfactory epithelium. Aox 2 exhibits the highest expression among Aox genes in the VNO [Colour figure can be viewed at wileyonlinelibrary.com] 
Next, we examined existing literature to assess whether the candidate genes may have an olfaction-related function. For Trpc2, S100z, Aox2, MsInl, olfaction-related functions have previously been suggested in the literature. The inactivation of Trpc2, an ion channel involved in vomeronasal signalling, has been previously described in several lineages with a reduced VNS and is well established (Liman \& Innan, 2003; Yohe et al., 2017; Yu et al., 2010). A VNS reduction associated inactivation of $\mathrm{S100z}$, Aox2 and MsInl has not been discussed before as far as we know. The function of $\mathrm{S100z}$ is largely uncharacterized. However, S100 proteins are calcium-binding proteins that may interact with ion channels and the $\mathrm{S} 100 \mathrm{z}$ ortholog in teleost fish exhibits an olfactory placode-specific expression (Hermann, Donato, Weiger, \& Chazin, 2012; Kraemer, Saraiva, \& Korsching, 2008). Aox 2 has been suggested to be involved in metabolizing of odorants in rodents (Kurosaki et al., 2004), and MsInl has been suggested to play a role in the evolution of olfaction in apes (Kim et al., 2012).

No existing studies suggest olfaction-related functions for Ubqln5, Tmprss11g and Kcnmb3, as far as we know. Ubqln5 has only recently been discovered based on sequence homology to Ubqln genes, which usually are related to protein degradation through the ubiquitin-proteasome system (Marin, 2014). Tmprss11g has been found to be differentially expressed in uterine glands (Kelleher, Burns, Behura, Wu, \& Spencer, 2016). So far there is no indication of a VNS or olfaction-related function for Tmprss11g. Kcnmb3 is a regulatory subunit of BK potassium channels and has been implicated in epilepsy in humans (Hu et al., 2003; Lorenz, Heils, Kasper, \& Sander, 2007), thus likely serves neuronal functions.

While all seven candidate gene losses may potentially have uncharacterized olfactory functions and could be associated with a reduction of the VNS, we focused on the four genes which are most likely to have a VNS or olfaction-related function based on their expression or based on knowledge from previous studies: Trpc2, S100z, Aox2 and Mslnl.

To obtain a more complete picture about the inactivation of the candidate genes in mammals, we investigated 9 additional genomes, considering a total of 115 placental mammalian genomes (Figure 4, Figure S3). To exclude the possibility of sequencing or assembly errors that can mimic real mutations, we validated inactivating mutations in all genes either by unassembled DNA sequencing reads or by the presence of shared mutations between independently assembled sister species genomes (Hecker et al., 2017; Hecker et al., 2019; Table S3 and Figures S4-S9). Furthermore, we found significant evidence that the candidate genes evolve under relaxed selection on the branches associated with species in which the gene was inactivated ( $p$-value $<10^{-4}$, Table S4; Wertheim et al., 2015), which further supports the inactivation the candidate genes. In contrast, Trpc2, S100z and Aox2 evolve largely under purifying selection ( $\Omega<0.12$ for $>85 \%$ of sites) along branches associated with species with intact VNS (Table S5). This is less pronounced for MsInl ( $\Omega<0.03$ for $\sim 65 \%$ of sites; Table S5). Together, this extensive manual validation confirmed that $\operatorname{Trpc} 2, \mathrm{S100z}$, Aox2 and MsInl are preferentially inactivated in mammals that have a reduced VNS.
In the following, we present a detailed analysis of these genes and discuss how their functions relate to the evolutionary reduction of the VNS or main olfactory system.

\section{3 | Inactivation of calcium signal transduction- related genes $\operatorname{Trpc} 2$ and $\mathrm{S} 100 \mathrm{z}$}

The VNO contains a layer of primary sensory neurons that are excited through a signalling cascade following stimulation by chemical cues (Francia, Pifferi, Menini, \& Tirindelli, 2014). This signalling cascade leads to a depolarization of the plasma membrane through influx of cations such as calcium, which is mediated by ion channels. Among other factors, proteins in the $\mathbf{S} 100$ family are involved in a variety of calcium-dependent processes and have also been linked to the regulation of calcium ion channels (van de Graaf et al., 2003; Hermann et al., 2012). Related to calcium-dependent processes, we detected the convergent loss of Trpc2 and S100z, a less well-characterized member of the $\mathbf{S 1 0 0}$ family, preferentially in species without a morphologically intact VNS.

The top candidate gene that was found in our screen is Trpc2, which encodes a transient cation channel that plays an important role for calcium signalling in primary sensory neurons of the VNO (Isogai et al., 2011; Lucas, Ukhanov, Leinders-Zufall, \& Zufall, 2003). Trpc2 knockout affects sociosexual behaviour in mice, although VNO function is not completely abolished (Kim, Ma, \& Yu, 2011; Stowers et al., 2002).

In our screen, Trpc2 was inactivated in all placental mammals with a reduced VNS whereas mammals that do not exhibit a regression of the VNS maintain an intact Trpc2 gene. The inactivation of Trpc2 has previously been associated with a nonfunctional VNO. With our study, we confirm and extend these previous findings that Trpc2 was inactivated in catarrhines, cetaceans and several bats (Liman \& Innan, 2003; Yohe et al., 2017; Yu et al., 2010). Our screen further showed that Trpc2 is inactivated in the aquatic manatee (Trichechus manatus) that also lacks a functional VNS. All mammals in our screen that do not exhibit a regression of the VNS maintain an intact Trpc2 gene with one exception. In the red deer (Cervus elaphus), which possesses a functional VNS, we found a confirmed frameshifting mutation in the last exon (Table S3); however, this mutation is far downstream of the last transmembrane domain therefore just leads to a different $\mathrm{C}$-terminal protein sequence (Figure S10). Such C-terminal alterations are a common evolutionary change in conserved genes (Sharma et al., 2016). Further supporting that Trpc2 is a functional gene in Cervus elaphus, we estimated Trpc2 to evolve under purifying selection in this species without any evidence for significantly relaxed selection rates ( $p$-value > .1; Table S4). Together, our analysis of 106 mammalian species provides a clear association between the convergent inactivation of Trpc2 and the convergent reduction of the VNS, suggesting that $\operatorname{Trpc} 2$ is a marker gene for the presence of the VNS.

Next, we analysed Trpc2 in sequenced mammals for which morphological VNS data are not available. We found that, in addition 


\section{\%intact reading frame}

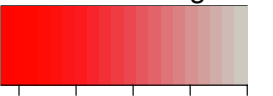

$20 \% \quad 40 \% \quad 60 \% \quad 80 \% 100 \%$

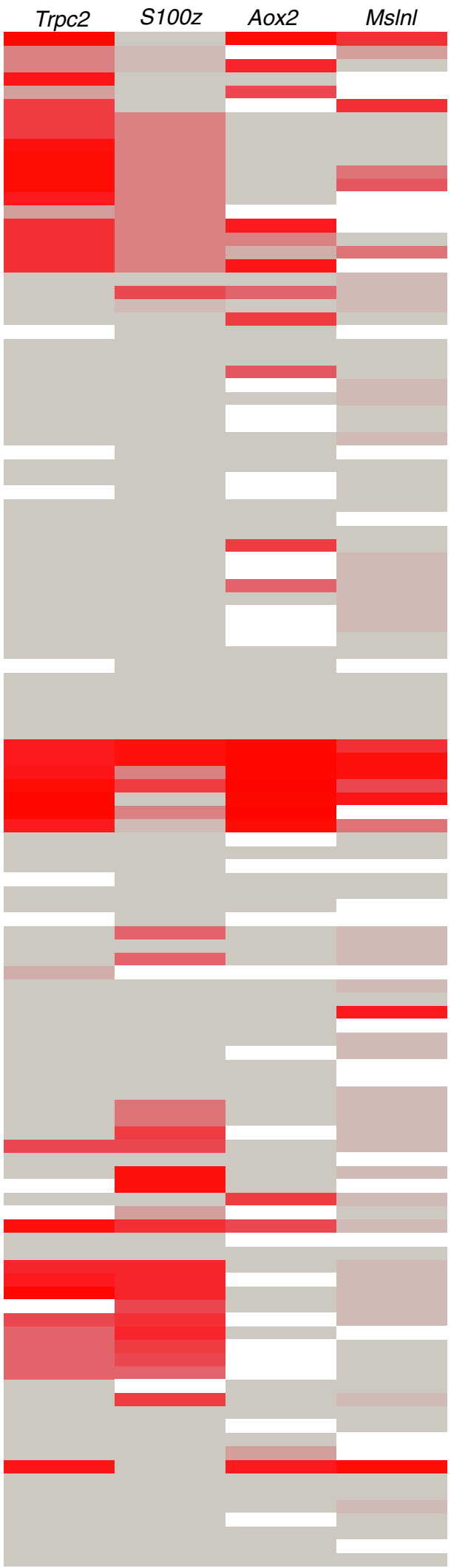

Homo sapiens dytes Gorilla gorilla Pongo pygmaeus Nomascus leucogenys Macaca mulatta Macaca fascicularis Papio anubis Mandrillus leucophaeus Cercocebus atys Chlorocebus sabaeus Nasalis larvatus

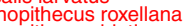
Colobus angolensi Piliocolobus tephrosceles Callithrix jacchus Aotus nancymaae Saimiri boliviensis Cebus capucinus Otolemur garnetti Microcebus murinus ropithecus coquereli Galeopterus variegatus Jupaia chinensis Microtus uch Cricetulus griseus Mesocricetus auratus Peromyscus maniculatus Mus caroli Mus pahari Meriones unguiculatus Nannospalax galili Castor canadensis Dipodomys ordii

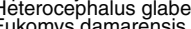
Cavia porcellus Cavia porcellus Octodon degus
Spermophilus tridecemlineatus Marmota marmota Oryctolagus cuniculus

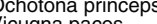
Camelus ferus Camelus bactrianus Camelus dromedarius Uursiops truncatus Delphinapterus leucas ipotes vexillifer Physeter catodon Balaenoptera acutorostrata Balaena mysticetus Bos taurus Bison bison ( Bubalus bubalis Ovis aries Ovis canadensis Capra hircus 作 quus caballus quus przewalsk Equus asinus Acimum elis catu Acinonyx jubatus Panthera pardus Canis lupus Lycaon pictus

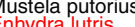
Ailurus fulgens Ailuropoda melanoleuca Ursus maritimus dobenus rosmarus 年 Manis pentadactyla Manis javanica Pteropus alecto Pteropus vampyrus Rhinolophus sinicus Hipposideros armiger Eptesicus fuscus Myotis davidii Myotis brandtii Miniopterus natalensis Desmodus rotundus Erinaceus europaeus Sorex araneus Condylura cristata oxodonta africana richechus manatus Chrysochloris asiatica Elephantulus edward Orycteropus afer Dasypus novemcinctus
Catarrhini

FIGURE 4 Presence or inactivation of VNS-associated candidate genes in 115 mammalian species. The colour gradient shows the intactness of the reading frame of a gene, computed as the percentage of the longest intact region of the reading frame for each species. Grey colours indicate an intact gene and red colours an inactivated gene. White areas indicate missing genomic data. Red font indicates species in which Trpc2 was inactivated. Based on our analysis, the inactivation of Trpc2, S100z, Aox2 and MsInl often occurred in the same lineages [Colour figure can be viewed at wileyonlinelibrary. com]

\section{Cetacea}

\section{Lutrinae}

Pinnipedia

Chiroptera

Sirenia 
to species with a reduced VNS, Trpc2 is only inactivated in several semi-aquatic mammals. Consistent with our analysis, a previous study showed that Trpc2 exhibits inactivating mutations in the semiaquatic river otter (Lontra) and harbour seal (Phoca), but not in sea lion (Zalophus californianus; Yu et al., 2010). Extending these findings, we identified a splice-site mutation in the sea otter (Enhydra lutris) Trpc2 gene and found Trpc2 to be under relaxed selection ( $p$ value $<.004$ ) in this species (Table S4). This indicates that the inactivation of Trpc2 is more widespread in Lutrinae. Our analysis also showed that Hawaiian monk seal (Neomonachus schauinslandii) and Weddell seal (Leptonychotes weddellii) share a premature stop codon in Trpc2 (Figure S6C). In contrast, Pacific walrus (Odobenus rosmarus divergens) contains an intact Trpc2 gene. Combined with the findings of Yu et al, this suggests that Trpc2 was inactivated in the common ancestor of Phocidae (Yu et al., 2010). Morphological characterization of the VNO and $A O B$ in seals, otters and walruses would be interesting for verifying the suitability of Trpc2 as a marker gene for the VNS (as discussed below).

The second-ranked gene that was discovered in our genomic screen is the calcium-binding $\mathrm{S100z}$ gene, whose convergent inactivation has not been described previously. While the molecular function of $\mathrm{S} 100 \mathrm{z}$ has not been studied in detail, S100 proteins generally participate in a variety of calcium-dependent processes that are important for neurons and the $\mathrm{S100z}$ ortholog in teleost fish was found to be specifically expressed in the olfactory placode (Kraemer et al., 2008). To further investigate whether $\mathrm{S} 100 \mathrm{z}$ may play a role in the mammalian VNS, we analysed available gene expression data of the olfactory epithelium and the VNO from mouse (Mus musculus) and compared the expression of all S100 genes. Supporting that $\mathrm{S} 100 \mathrm{z}$ plays a role in VNO function, our analysis showed that $\mathrm{S} 100 \mathrm{z}$ exhibits the second highest expression in the VNO compared with other S100 genes and has a substantially higher expression in the VNO than in the olfactory epithelium (Figure 3a). Our analysis shows that the $\mathrm{S100z}$ gene of all Old World monkeys and Nancy Ma's night monkey (Aotus nancymaae) contains inactivating mutations, but not the $\mathrm{S} 100 \mathrm{z}$ gene of apes. However, we estimated $\mathrm{S} 100 \mathrm{z}$ to evolve neutrally $\left(p\right.$-value $<10^{-8}$ ) in apes, which indicates that $\mathrm{S100z}$ is nonfunctional in Catarrhini (Table S4). Although we did not identify inactivating mutations in the manatee (Trichechus manatus), we estimated that $\mathrm{S100z}$ also evolves neutrally ( $p$-value $<10^{-3}$ ) in this species (Table S4). All cetaceans share a mutation of the ATG start codon, which suggests that $\mathrm{S} 100 \mathrm{z}$ was already inactivated in the cetacean stem lineage (Figure S7C). Of all analysed cetacean species, only the sperm whale (Physeter catodon) exhibits an in-frame ATG further upstream of the ancestral ATG codon, which might serve as a new start codon. In addition, $\mathrm{S100z}$ was inactivated in bats and Phocidae species, but not Pacific walrus (Odobenus rosmarus divergens).

Interestingly, we found $\mathrm{S} 100 \mathrm{z}$ to be inactivated in the sanguivorous common vampire bat (Desmodus rotundus) which contains an intact VNS. To investigate whether the loss of $\mathrm{S100z}$ also occurred in additional bat species that are not part of our screen, we examined nine additional bat genomes for inactivating mutations. Based on species for which we could properly align a sufficient amount of sequences for the genes, we found that both Trpc2 and S100z are intact in all Phyllostomidae species, which have an intact VNS. Both genes are inactivated in all Pteropodidae species and Noctilio leporinus which lack a functional VNS (Table S6). Whether the specific loss of $\mathrm{S} 100 \mathrm{z}$ in Desmodus rotundus is associated with olfactory changes or is compensated otherwise remains an open question.

The $\mathrm{S} 100 \mathrm{z}$ gene of several additional Caniformia species including Enhydra lutris contains inactivating mutations, which could be further evidence for a nonfunctional VNS in Lutrinae. In contrast, all analysed Felidae species carry an intact $\mathrm{S} 100 \mathrm{z}$ gene. White-tailed deer (Odocoileus virginianus) has an inactivated $\mathrm{S} 100 \mathrm{z}$ gene, and goat (Capra hircus) contains a frameshifting mutation in the last exon. Nonetheless, the inactivation of and relaxed selection rates on $\mathrm{S} 100 \mathrm{z}$ overlaps with a reduced VNS and the inactivation of Trpc2.

\section{4 | Odorant-related inactivation of aldehyde oxidase gene Aox2}

Perception of pheromones or other chemical cues relies on the stimulation of specialized receptors by chemical compounds, referred to as odorants which may include pheromones. To terminate or reduce the duration of stimuli, odorants are degraded or inactivated by enzymes such as aldehyde oxidases. Aox2 (also known as Aoh3 or Aox3/1) has been found to be expressed in the Bowman's glands of olfactory epithelium of rodents and has been suggested to be involved in the oxidation of odorants (Kurosaki et al., 2004). In addition, functional studies of the Aox 2 ortholog in invertebrates suggest that Aox 2 plays a role in pheromone degradation (Choo, Pelletier, Atungulu, \& Leal, 2013; Pelletier et al., 2007). Ranked third in our screen, we identified Aox 2 as another gene that is preferentially lost in mammals lacking a functional VNS.

We analysed the expression of all Aox family members using the mouse gene expression data of the olfactory epithelium and the VNO. Our analysis shows that while Aox 2 has a noticeably higher expression in the olfactory epithelium than in the VNO, it is clearly expressed in the VNO. Moreover, no other Aox gene has a higher expression in the VNO (Figure 3). This could suggest that Aox2 has an odorant-degrading function in the vomeronasal organ, as well as in the olfactory epithelium of the main olfactory system.

Our analysis of 115 mammalian genomes shows that Aox 2 is lost in all fully aquatic mammals (cetaceans and Trichechus manatus), the semi-aquatic pinnipeds and several primates, but not in Cercopithecidae species. Previous studies described only the inactivation of Aox 2 in primates and common bottlenose dolphin (Tursiops truncatus) and did not associate it with VNS reduction (Kurosaki et al., 2013). We also identified splice-site mutations in two rodents, Damaraland mole-rat (Fukomys damarensis) and American beaver (Castor canadensis). The splice-site mutation in Fukomys damarensis is likely polymorphic (Table S3), and we estimated only marginally relaxed selection rates ( $p$-value <.04) for Aox 2 in this species (Table S4). In contrast, no evidence for relaxed selection rates ( $p$-value $>.5$ ) 
was found for Aox2 in American beaver (Castor canadensis). Given that Aox 2 was estimated to evolve largely under purifying selection in both species, the gene may still be functional in both rodents (Table S4). Together, our results suggest that the inactivation of Aox2 largely coincides with mammals that do not possess a functional VNS. Given its expression in the VNO, Aox2 poses an interesting candidate for a convergently inactivated gene associated with convergent VNS reduction.

\section{5 | Inactivation of olfactory epithelium and VNO- specific gene MsInl}

Given that the function of MsInl has not been experimentally characterized, we analysed its expression. This analysis showed that MsInl is expressed in both olfactory epithelium and VNO of mouse (Mus musculus, Figure 3c). Consistent with this finding, a previous study suggested that the loss of MsInl is linked to the evolution of olfaction in apes based on its expression in olfactory epithelium (Kim et al., 2012). Our study confirms that Mlsnl was inactivated in several primates including Old World monkeys, except for Papionini species. Furthermore, we detected that MsInl was inactivated in all fully aquatic mammals (cetaceans and Trichechus manatus). We further found that all mammals that do not show a VNS reduction have an intact MsInl gene with the exception of the Przewalski's horse (Equus przewalskii). The MsInl gene in Equus przewalskii contains a frameshifting mutation in exon 8 (Figure S13). Hence, MsInl appears to be lost almost exclusively in mammals that do not possess a functional VNS. Future studies may elucidate whether Mslnl is involved in VNS specific functions.

\section{4 | DISCUSSION}

Using a forward genomics approach, our screen detected that the convergent inactivation of Trpc2, S100z, Aox2 and MInsl coincides with the convergent reduction of the VNS in Catarrhini, Cetacea, Chiroptera and Sirenia (Figures 2 and 4). While Trpc2 and S100z are preferentially expressed within the VNO suggesting VNO-specific roles, Aox 2 and MsInl likely play a role for both the VNS and the main olfactory system. In particular, an odorant-degrading function of Aox 2 is likely important for both olfactory systems. Our findings that genes which are likely important for both olfactory systems were convergently lost in VNS-reduced mammals is in agreement with the co-occurring reduction of the VNS and main olfactory system in different mammalian lineages. In cetaceans, the loss of the VNO occurred at the same time during evolution as the reduction of the olfactory bulb (Kishida, Thewissen, Hayakawa, Imai, \& Agata, 2015). Hence, the inactivation of Aox2 and MsInl, in particular, is likely a genomic signature of the reduction of both the VNS and the main olfactory system.

Our screen does not only exemplify the utility of the combination of morphology and comparative genomics for understanding associations between phenotypic and genomic changes, but also shows that trait and gene losses can be associated with specific ecological settings during mammalian evolution. In particular, the convergent reduction of the VNS during mammalian evolution and the associated inactivation of Trpc2, S100z, Aox2 and MsInl happened in different ecological niches and likely occurred for different reasons. A general reduction of both olfactory systems as a consequence of the transition to an aquatic habitat is likely an explanation for relaxed selection and inactivation of the candidate genes in fully aquatic mammals. A semi-aquatic lifestyle may also coincide with a reduced sense of olfaction and vomeronasal system in the semi-aquatic pinnipeds and otters which could explain the inactivation of $\operatorname{Trpc2}$, $\mathrm{S} 100 \mathrm{z}$ and Aox2 in these species. Several mammalian species may have substituted sociosexual functions of the VNS by other senses or changes in social behaviour. For apes and Old World monkeys, sociosexual functions of the VNS might have become less important after the evolution of trichromatic colour vision (Yu et al., 2010), which is supported by a simultaneous occurrence of colour vision and relaxed selection pressure on the Trpc2 gene in the common ancestor of Cercopithecidae and Hominoidea (Liman \& Innan, 2003). For example, mating behaviour in chimpanzees and baboons is influenced by optical cues such as prominent reddish skin swellings that indicate the follicular phase (Dixson, 2015). Also, the evolution of vocal communication might have contributed to a decrease of selection pressure on the VNS. Songs of humpback whales (Megaptera novaeangliae), for instance, have been suggested to have an intersexual function (Smith, Goldizen, Dunlop, \& Noad, 2008). It is less clear why certain bats exhibit a reduced VNS. In this context, it has been suggested that the main olfactory system might compensate for the lack of a functional VNS (Yohe et al., 2017). If this is the case, overlapping functions between the VNS and main olfactory system might also explain why most bats maintained an intact Aox2 and MsInl gene, in contrast to cetaceans and several primates. Understanding the evolutionary triggers that cause VNS reduction in different mammalian lineages in their entirety will remain a challenge. However, lineagespecific evolutionary scenarios such as the evolution of colour vision in catarrhines and the change to an aquatic habitat for cetaceans and semi-aquatic species provide likely explanations for the relaxed selection on the VNS and main olfactory system, which permitted the inactivation of Trpc2, S100z, Aox2 and MsInl.

Our analysis of 115 mammalian species confirmed the inactivation of Trpc2 as a clear marker for VNS reduction and supports the idea that in some cases molecular marker genes can be useful for predicting phenotypes from genomic data. Trpc2 is inactivated in all mammals with a reduced VNS, while a functional Trpc2 gene is present in all examined mammals with a morphologically intact VNS. Yohe and Davalos demonstrated that Trpc2 selection rates have the potential to predict the presence or absence of a functional VNS in bats (Yohe \& Davalos, 2018). Based on our results, Trpc2 inactivation may be suitable for predicting the presence or absence of a functional VNS in mammals in general. This could be useful for inferring VNS function from newly sequenced genomes of less characterized mammals in future. For example, extending a previous study (Yu et al., 2010), we detected the inactivation of Trpc2 in 
additional Phocidae and Lutrinae species, for which no morphological data about the presence of a functional VNO or AOB are available. Our findings that Phocidae have lost $\mathrm{S} 100 \mathrm{z}$ and $\mathrm{Aox} 2$ provide additional genomic evidence that this lineage may not possess a functional VNS. Interestingly, the related Pacific walrus (Odobenus rosmarus divergens) maintains intact Trpc2 and $\mathrm{S} 100 \mathrm{z}$ genes. In sea otter (Enhydra lutris), a member of Lutrinae, Trpc2 and S100z are inactivated. Whether the semi-aquatic Phocidae and Lutrinae species maintain functional vomeronasal systems is not known but could be addressed in future studies. Together, these findings make Phocidae and Lutrinae species attractive targets for the morphological characterization of the VNO and AOB, which would help to assess whether molecular marker genes can be used for predicting the presence or absence of the VNS.

Apart from using these marker genes for predicting the presence/absence of the VNS in mammalian species, they can be used for analyses of selective pressures on the VNS under different ecological settings. Phylogenetic and/or population genetic concepts are suited to estimate the strength of selection on genes as an indirect way to analyse selection pressure on the associated trait as a proxy for its biological significance. For instance, the hypothesis that a semi-aquatic lifestyle is associated with a nonfunctional VNO in Phocidae and Lutrinae can be tested by selection pressure analyses in mammals of different phylogenetic lineages. This will provide insights into the extent to which VNS functionality is associated to a semi-aquatic lifestyle. These hypotheses may preferentially be tested in semi-aquatic species with strictly terrestrial congeners such as water shrews (Neomys fodiens, N. anomalus) as representatives of the Order Eulipotyphla or beavers (Castor spec.) and muskrats (Ondatra zibethicus) as representatives of the Order Rodentia.

Overall, with this study, we not only extend previous findings and provide new insights into the genomic changes that are associated with the convergent reduction of the VNS in placental mammals. We further exemplify that loci identified within the conceptual framework of comparative genomics (e.g. forward genomics approaches) uncover marker genes i) to predict phenotypes from genomic data and ii) to help estimating the biological significance of these phenotypes in different ecological settings.

\section{ACKNOWLEDGEMENTS}

We thank the computer facilities of the Max Planck Institute of Molecular Cell Biology and Genetics and the Max Planck Institute for the Physics of Complex Systems for their support and for providing IT infrastructure. We further thank Moritz Blumer and David Jebb for preparing the additional nine bat genomes for analysis. This work was supported by the Max Planck Society and the Leibniz Association (SAW-2016-SGN-2).

\section{CONFLICT OF INTEREST}

The authors declare that no conflicts of interest exist.

\section{AUTHOR CONTRIBUTIONS}

All authors contributed to the study design. N.H. performed the computational analysis and drafted the manuscript. U.L. characterized the presence and absence of the VNS through literature research and inspection of zoological collections. All authors revised and approved the manuscript.

\section{DATA AVAILABILITY STATEMENT}

All analysed genome assemblies (Table S1) are publicly available at the UCSC genome browser, NCBI GenBank or the provided URL. DNA sequencing read data (Table S3) are publicly available at the NCBI Trace and Sequence Read Archive. The analysed RNAseq data are publicly available at the ArrayExpress.

\section{ORCID}

\author{
Nikolai Hecker (iD https://orcid.org/0000-0003-1693-4257 \\ Heiko Stuckas (iD https://orcid.org/0000-0002-5690-0994 \\ Michael Hiller (iD https://orcid.org/0000-0003-3024-1449
}

\section{REFERENCES}

Albalat, R., \& Canestro, C. (2016). Evolution by gene loss. Nature Reviews Genetics, 17, 379-391. https://doi.org/10.1038/nrg.2016.39

Bean, N. J., \& Wysocki, C. J. (1989). Vomeronasal organ removal and female mouse aggression: The role of experience. Physiology \& Behavior, 45, 875-882. https://doi.org/10.1016/0031-9384(89)90209-6

Bertmar, G. (1969). The vertebrate nose, remarks on its structural and functional adaptation and evolution. Evolution, 23, 131-152.

Bhatnagar, K. P., \& Meisami, E. (1998). Vomeronasal organ in bats and primates: Extremes of structural variability and its phylogenetic implications. Microscopy Research and Technique, 43, 465-475. https:// doi.org/10.1002/(SICI)1097-0029(19981215)43:6<465:AID-JEMT1 $>3.0 . \mathrm{CO} ; 2-1$

Blanchette, M., Kent, W. J., Riemer, C., Elnitski, L., Smit, A. F., Roskin, K. M., ... Miller, W. (2004). Aligning multiple genomic sequences with the threaded blockset aligner. Genome Research, 14, 708-715. https ://doi.org/10.1101/gr.1933104

Burton, P. R. (1990). Vomeronasal and olfactory nerves of adult and larval bullfrogs: II. Axon terminations and synaptic contacts in the accessory olfactory bulb. The Journal of Comparative Neurology, 292, 624-637. https://doi.org/10.1002/cne.902920411

Burton, P. R., Coogan, M. M., \& Borror, C. A. (1990). Vomeronasal and olfactory nerves of adult and larval bullfrogs: I. Axons and the distribution of their glomeruli. The Journal of Comparative Neurology, 292, 614-623. https://doi.org/10.1002/cne.902920410

Chikina, M., Robinson, J. D., \& Clark, N. L. (2016). Hundreds of genes experienced convergent shifts in selective pressure in marine mammals. Molecular Biology and Evolution, 33, 2182-2192. https://doi. org $/ 10.1093 / \mathrm{molbev} / \mathrm{msw} 112$

Choo, Y. M., Pelletier, J., Atungulu, E., \& Leal, W. S. (2013). Identification and characterization of an antennae-specific aldehyde oxidase from the navel orangeworm. PLoS ONE, 8, e67794. https://doi. org/10.1371/journal.pone.0067794

D'Aniello, B., Semin, G. R., Scandurra, A., \& Pinelli, C. (2017). The vomeronasal organ: A neglected organ. Frontiers in Neuroanatomy, 11, 70. https://doi.org/10.3389/fnana.2017.00070 
Diez-Roux, G., Banfi, S., Sultan, M., Geffers, L., Anand, S., Rozado, D., .. Ballabio, A. (2011). A high-resolution anatomical atlas of the transcriptome in the mouse embryo. PLoS Biology, 9, e1000582. https:// doi.org/10.1371/journal.pbio.1000582

Dixson, A. (2015). Primate sexuality. In The International Encyclopedia of human sexuality (pp. 861-1042). Hoboken, NJ: John Wiley \& Sons.

Doving, K. B., \& Trotier, D. (1998). Structure and function of the vomeronasal organ. Journal of Experimental Biology, 201, 2913-2925.

Dulac, C. (2000). Sensory coding of pheromone signals in mammals. Current Opinion in Neurobiology, 10, 511-518. https://doi. org/10.1016/S0959-4388(00)00121-5

Ehrlich, F., Fischer, H., Langbein, L., Praetzel-Wunder, S., Ebner, B., Figlak, K., ... Eckhart, L. (2019). Differential evolution of the epidermal keratin cytoskeleton in terrestrial and aquatic mammals. Molecular Biology and Evolution, 36, 328-340. https://doi.org/10.1093/molbe $\mathrm{v} / \mathrm{msy} 214$

El-Gebali, S., Mistry, J., Bateman, A., Eddy, S. R., Luciani, A., Potter, S. C., ... Finn, R. D. (2019). The Pfam protein families database in 2019. Nucleic Acids Research, 47, D427-D432. https://doi.org/10.1093/nar/ gky995

Felsenstein, J. (1973). Maximum-likelihood estimation of evolutionary trees from continuous characters. American Journal of Human Genetics, 25, 471-492.

Francia, S., Pifferi, S., Menini, A., \& Tirindelli, R. (2014). Vomeronasal receptors and signal transduction in the vomeronasal organ of mammals. In C. Mucignat-Caretta (Ed.), Neurobiology of chemical communication (pp. 297-324). Boca Raton, FL: CRC Press, Taylor \& Francis Group.

Gonzalez, A., Morona, R., Lopez, J. M., Moreno, N., \& Northcutt, R. G. (2010). Lungfishes, like tetrapods, possess a vomeronasal system. Frontiers in Neuroanatomy, 4. https://doi.org/10.3389/ fnana.2010.00130

Grus, W. E., \& Zhang, J. (2009). Origin of the genetic components of the vomeronasal system in the common ancestor of all extant vertebrates. Molecular Biology and Evolution, 26, 407-419. https://doi. org $/ 10.1093 / \mathrm{molbev} / \mathrm{msn} 262$

Haeussler, M., Zweig, A. S., Tyner, C., Speir, M. L., Rosenbloom, K. R., Raney, B. J., ... Kent, W. J. (2019). The UCSC Genome Browser database: 2019 update. Nucleic Acids Research, 47, D853-D858. https:// doi.org/10.1093/nar/gky1095

Harris, R. S. (2007). Improved pairwise alignment of genomic DNA. PhD, Pennsylvania State University, PA, USA.

Hecker, N., Sharma, V., \& Hiller, M. (2017). Transition to an aquatic habitat permitted the repeated loss of the pleiotropic KLK8 gene in mammals. Genome Biology and Evolution, 9, 3179-3188. https://doi. org/10.1093/gbe/evx239

Hecker, N., Sharma, V., \& Hiller, M. (2019). Convergent gene losses illuminate metabolic and physiological changes in herbivores and carnivores. Proceedings of the National Academy of Sciences of the United States of America, 116, 3036-3041. https://doi.org/10.1073/ pnas.1818504116

Hermann, A., Donato, R., Weiger, T. M., \& Chazin, W. J. (2012). S100 calcium binding proteins and ion channels. Frontiers in Pharmacology, 3 , 67. https://doi.org/10.3389/fphar.2012.00067

Hiller, M., Schaar, B. T., Indjeian, V. B., Kingsley, D. M., Hagey, L. R., \& Bejerano, G. (2012). A "forward genomics" approach links genotype to phenotype using independent phenotypic losses among related species. Cell Reports, 2, 817-823. https://doi.org/10.1016/j. celrep.2012.08.032

Hu, S., Labuda, M. Z., Pandolfo, M., Goss, G. G., McDermid, H. E., \& Ali, D. W. (2003). Variants of the KCNMB3 regulatory subunit of maxi BK channels affect channel inactivation. Physiological Genomics, 15, 191-198. https://doi.org/10.1152/physiolgenomics.00110.2003

Huelsmann, M., Hecker, N., Springer, M. S., Gatesy, J., Sharma, V., \& Hiller, M. (2019). Genes lost during the transition from land to water in cetaceans highlight genomic changes involved in aquatic adaptations. bioRxiv. https://doi.org/10.1101/521617

Isogai, Y., Si, S., Pont-Lezica, L., Tan, T., Kapoor, V., Murthy, V. N., \& Dulac, C. (2011). Molecular organization of vomeronasal chemoreception. Nature, 478, 241-245. https://doi.org/10.1038/nature10437

Jiang, P., Josue, J., Li, X., Glaser, D., Li, W., Brand, J. G., ... Beauchamp, G. K. (2012). Major taste loss in carnivorous mammals. Proceedings of the National Academy of Sciences of the United States of America, 109, 4956-4961. https://doi.org/10.1073/pnas.1118360109

Kelleher, A. M., Burns, G. W., Behura, S., Wu, G., \& Spencer, T. E. (2016). Uterine glands impact uterine receptivity, luminal fluid homeostasis and blastocyst implantation. Scientific Reports, 6, 38078.

Kelliher, K. R. (2007). The combined role of the main olfactory and vomeronasal systems in social communication in mammals. Hormones and Behavior, 52, 561-570. https://doi.org/10.1016/j.yhbeh.2007.08.012

Kent, W. J., Baertsch, R., Hinrichs, A., Miller, W., \& Haussler, D. (2003). Evolution's cauldron: Duplication, deletion, and rearrangement in the mouse and human genomes. Proceedings of the National Academy of Sciences of the United States of America, 100, 11484-11489.

Kim, D. W., Jeong, S., Kim, D. S., Kim, H. S., Seo, S. B., \& Hahn, Y. (2012). Inactivation of the MSLNL gene encoding mesothelin-like protein during African great ape evolution. Gene, 496, 17-21. https://doi. org/10.1016/j.gene.2012.01.005

Kim, S., Cho, Y. S., Kim, H. M., Chung, O., Kim, H., Jho, S., ... Yeo, J. H. (2016). Comparison of carnivore, omnivore, and herbivore mammalian genomes with a new leopard assembly. Genome Biology, 17, 211.

Kim, S., Ma, L., \& Yu, C. R. (2011). Requirement of calcium-activated chloride channels in the activation of mouse vomeronasal neurons. Nature Communications, 2, 365. https://doi.org/10.1038/ncomm s1368

Kishida, T., Kubota, S., Shirayama, Y., \& Fukami, H. (2007). The olfactory receptor gene repertoires in secondary-adapted marine vertebrates: Evidence for reduction of the functional proportions in cetaceans. Biology Letters, 3, 428-430. https://doi.org/10.1098/rsbl.2007.0191

Kishida, T., Thewissen, J., Hayakawa, T., Imai, H., \& Agata, K. (2015). Aquatic adaptation and the evolution of smell and taste in whales. Zoological Letters, 1, 9. https://doi.org/10.1186/s40851-014-0002-z

Kodama, Y., Shumway, M., \& Leinonen, R. \& International Nucleotide Sequence Database Collabration (2012). The sequence read archive: Explosive growth of sequencing data. Nucleic Acids Research, 40, D54-D56. https://doi.org/10.1093/nar/gkr854

Kolesnikov, N., Hastings, E., Keays, M., Melnichuk, O., Tang, Y. A., Williams, E., ... Brazma, A. (2015). ArrayExpress update-simplifying data submissions. Nucleic Acids Research, 43, D1113-D1116. https:// doi.org/10.1093/nar/gku1057

Kraemer, A. M., Saraiva, L. R., \& Korsching, S. I. (2008). Structural and functional diversification in the teleost S100 family of calcium-binding proteins. BMC Evolutionary Biology, 8, 48. https://doi. org/10.1186/1471-2148-8-48

Kurosaki, M., Bolis, M., Fratelli, M., Barzago, M. M., Pattini, L., Perretta, G., ... Garattini, E. (2013). Structure and evolution of vertebrate aldehyde oxidases: From gene duplication to gene suppression. Cellular and Molecular Life Sciences, 70, 1807-1830. https://doi.org/10.1007/ s00018-012-1229-5

Kurosaki, M., Terao, M., Barzago, M. M., Bastone, A., Bernardinello, D., Salmona, M., \& Garattini, E. (2004). The aldehyde oxidase gene cluster in mice and rats. Aldehyde oxidase homologue 3 , a novel member of the molybdo-flavoenzyme family with selective expression in the olfactory mucosa. Journal of Biological Chemistry, 279, 50482-50498.

Liman, E. R., \& Innan, H. (2003). Relaxed selective pressure on an essential component of pheromone transduction in primate evolution. Proceedings of the National Academy of Sciences of the United States of America, 100, 3328-3332. https://doi.org/10.1073/pnas.06361 23100 
Liu, Z., Liu, G., Hailer, F., Orozco-terWengel, P., Tan, X., Tian, J., ... Li, M. (2016). Dietary specialization drives multiple independent losses and gains in the bitter taste gene repertoire of Laurasiatherian Mammals. Frontiers in Zoology, 13, 28. https://doi.org/10.1186/ s12983-016-0161-1

Lopes-Marques, M., Ruivo, R., Alves, L. Q., Sousa, N., Machado, A. M., \& Castro, L. F. C. (2019). The Singularity of Cetacea behavior parallels the complete inactivation of melatonin gene modules. Genes (Basel), 10, 121. https://doi.org/10.3390/genes10020121

Lorenz, S., Heils, A., Kasper, J. M., \& Sander, T. (2007). Allelic association of a truncation mutation of the KCNMB3 gene with idiopathic generalized epilepsy. American Journal of Medical Genetics. Part B: Neuropsychiatric Genetics, 144B, 10-13. https://doi.org/10.1002/ ajmg.b.30369

Loytynoja, A., \& Goldman, N. (2008). Phylogeny-aware gap placement prevents errors in sequence alignment and evolutionary analysis. Science, 320, 1632-1635. https://doi.org/10.1126/science.1158395

Lucas, P., Ukhanov, K., Leinders-Zufall, T., \& Zufall, F. (2003). A diacylglycerol-gated cation channel in vomeronasal neuron dendrites is impaired in TRPC2 mutant mice: Mechanism of pheromone transduction. Neuron, 40, 551-561. https://doi.org/10.1016/S0896-6273(03)00675-5

Mackay-Sim, A., Duvall, D., \& Graves, B. M. (1985). The West Indian manatee (Trichechus manatus) lacks a vomeronasal organ. Brain, Behavior and Evolution, 27, 186-194.

Marin, I. (2014). The ubiquilin gene family: Evolutionary patterns and functional insights. BMC Evolutionary Biology, 14, 63. https://doi. org/10.1186/1471-2148-14-63

Meisami, E., \& Bhatnagar, K. P. (1998). Structure and diversity in mammalian accessory olfactory bulb. Microscopy Research and Technique, 43, 476-499. https://doi.org/10.1002/(SICl)1097-0029(19981 215)43:6<476:AID-JEMT2>3.3.CO;2-M

Meredith, M. (2001). Human vomeronasal organ function: A critical review of best and worst cases. Chemical Senses, 26, 433-445. https:// doi.org/10.1093/chemse/26.4.433

Merkin, J., Russell, C., Chen, P., \& Burge, C. B. (2012). Evolutionary dynamics of gene and isoform regulation in Mammalian tissues. Science, 338, 1593-1599. https://doi.org/10.1126/science.1228186

Nery, M. F., Arroyo, J. I., \& Opazo, J. C. (2014). Increased rate of hair keratin gene loss in the cetacean lineage. BMC Genomics, 15, 869. https ://doi.org/10.1186/1471-2164-15-869

Niimura, Y., \& Nei, M. (2007). Extensive gains and losses of olfactory receptor genes in mammalian evolution. PLoS ONE, 2, e708. https:// doi.org/10.1371/journal.pone.0000708

Oelschläger, H. A., Buhl, E. H., \& Dann, J. F. (1987). Development of the nervus terminalis in mammals including toothed whales and humans. Annals of the New York Academy of Sciences, 519, 447-464. https:// doi.org/10.1111/j.1749-6632.1987.tb36316.x

Partha, R., Chauhan, B. K., Ferreira, Z., Robinson, J. D., Lathrop, K., Nischal, K. K., ... Clark, N. L. (2017). Subterranean mammals show convergent regression in ocular genes and enhancers, along with adaptation to tunneling. Elife, 6. https://doi.org/10.7554/eLife.25884.001

Pelletier, J., Bozzolan, F., Solvar, M., Francois, M. C., Jacquin-Joly, E., \& Maibeche-Coisne, M. (2007). Identification of candidate aldehyde oxidases from the silkworm Bombyx mori potentially involved in antennal pheromone degradation. Gene, 404, 31-40. https://doi. org/10.1016/j.gene.2007.08.022

Pfister, P., \& Rodriguez, I. (2005). Olfactory expression of a single and highly variable $\mathrm{V} 1 \mathrm{r}$ pheromone receptor-like gene in fish species. Proceedings of the National Academy of Sciences of the United States of America, 102, 5489-5494. https://doi.org/10.1073/pnas.04025 81102

Pond, S. L., Frost, S. D., \& Muse, S. V. (2005). HYPHY: Hypothesis testing using phylogenies. Bioinformatics, 21, 676-679. https://doi. org/10.1093/bioinformatics/bti079
Prudent, X., Parra, G., Schwede, P., Roscito, J. G., \& Hiller, M. (2016). Controlling for phylogenetic relatedness and evolutionary rates improves the discovery of associations between species' phenotypic and genomic differences. Molecular Biology and Evolution, 33, 21352150. https://doi.org/10.1093/molbev/msw098

Rodriguez, J. M., Rodriguez-Rivas, J., Di Domenico, T., Vazquez, J., Valencia, A., \& Tress, M. L. (2018). APPRIS 2017: Principal isoforms for multiple gene sets. Nucleic Acids Research, 46, D213-D217. https ://doi.org/10.1093/nar/gkx997

Schluter, D., Price, T., Mooers, A. O., \& Ludwig, D. (1997). Likelihood of ancestor states in adaptive radiation. Evolution, 51, 1699-1711. https ://doi.org/10.1111/j.1558-5646.1997.tb05095.x

Sharma, V., Elghafari, A., \& Hiller, M. (2016). Coding exon-structure aware realigner (CESAR) utilizes genome alignments for accurate comparative gene annotation. Nucleic Acids Research, 44, e103. https ://doi.org/10.1093/nar/gkw210

Sharma, V., Hecker, N., Roscito, J. G., Foerster, L., Langer, B. E., \& Hiller, M. (2018). A genomics approach reveals insights into the importance of gene losses for mammalian adaptations. Nature Communications, 9, 1215. https://doi.org/10.1038/s41467-018-03667-1

Sharma, V., Schwede, P., \& Hiller, M. (2017). CESAR 2.0 substantially improves speed and accuracy of comparative gene annotation. Bioinformatics, 33, 3985-3987. https://doi.org/10.1093/bioinforma tics/btx527

Smith, C. M., Hayamizu, T. F., Finger, J. H., Bello, S. M., McCright, I. J., Xu, J., ... Ringwald, M. (2019). The mouse Gene Expression Database (GXD): 2019 update. Nucleic Acids Research, 47, D774-D779. https:// doi.org/10.1093/nar/gky922

Smith, J. N., Goldizen, A. W., Dunlop, R. A., \& Noad, M. J. (2008). Songs of male humpback whales, Megaptera novaeangliae, are involved in intersexual interactions. Animal Behavior, 76, 467-477.

Stoddart, D. M. (1980). The ecology of vertebrate olfaction. New York, NY: Chapman and Hall.

Stowers, L., Holy, T. E., Meister, M., Dulac, C., \& Koentges, G. (2002). Loss of sex discrimination and male-male aggression in mice deficient for TRP2. Science, 295, 1493-1500. https://doi.org/10.1126/scien ce.1069259

Stowers, L., \& Marton, T. F. (2005). What is a pheromone? Mammalian pheromones reconsidered. Neuron, 46, 699-702. https://doi. org/10.1016/j.neuron.2005.04.032

Suarez, H. G., Langer, B. E., Ladde, P., \& Hiller, M. (2017). chainCleaner improves genome alignment specificity and sensitivity. Bioinformatics, 33, 1596-1603. https://doi.org/10.1093/bioinformatics/btx024

Suarez, R., Garcia-Gonzalez, D., \& de Castro, F. (2012). Mutual influences between the main olfactory and vomeronasal systems in development and evolution. Frontiers in Neuroanatomy, 6, 50. https://doi. org/10.3389/fnana.2012.00050

Tachikawa, K. S., Yoshihara, Y., \& Kuroda, K. O. (2013). Behavioral transition from attack to parenting in male mice: A crucial role of the vomeronasal system. Journal of Neuroscience, 33, 5120-5126. https ://doi.org/10.1523/JNEUROSCI.2364-12.2013

van de Graaf, S. F., Hoenderop, J. G., Gkika, D., Lamers, D., Prenen, J., Rescher, U., ... Bindels, R. J. (2003). Functional expression of the epithelial $\mathrm{Ca}(2+)$ channels (TRPV5 and TRPV6) requires association of the S100A10-annexin 2 complex. EMBO Journal, 22, 1478-1487. https://doi.org/10.1093/emboj/cdg162

Wang, R. T., \& Halpern, M. (1980). Light and electron microscopic observations on the normal structure of the vomeronasal organ of garter snakes. Journal of Morphology, 164, 47-67. https://doi.org/10.1002/ jmor.1051640105

Wertheim, J. O., Murrell, B., Smith, M. D., Kosakovsky Pond, S. L., \& Scheffler, K. (2015). RELAX: Detecting relaxed selection in a phylogenetic framework. Molecular Biology and Evolution, 32, 820-832. https://doi.org/10.1093/molbev/msu400 
Wittmer, C., \& Nowack, C. (2017). Epithelial crypts: A complex and enigmatic olfactory organ in African and South American lungfish (Lepidosireniformes, Dipnoi). Journal of Morphology, 278, 791-800. https://doi.org/10.1002/jmor.20673

Yohe, L. R., Abubakar, R., Giordano, C., Dumont, E., Sears, K. E., Rossiter, S. J., \& Davalos, L. M. (2017). Trpc2 pseudogenization dynamics in bats reveal ancestral vomeronasal signaling, then pervasive loss. Evolution, 71, 923-935.

Yohe, L. R., \& Davalos, L. M. (2018). Strength of selection on the Trpc2 gene predicts accessory olfactory bulb form in bat vomeronasal evolution. Biological Journal of the Linnean Society, 123, 796-804. https:// doi.org/10.1093/biolinnean/bly015

Young, J. M., Massa, H. F., Hsu, L., \& Trask, B. J. (2010). Extreme variability among mammalian V1R gene families. Genome Research, 20, 10-18. https://doi.org/10.1101/gr.098913.109

Yu, C. R. (2015). TRICK or TRP? What Trpc2(-/-) mice tell us about vomeronasal organ mediated innate behaviors. Frontiers in Neuroscience, 9, 221. https://doi.org/10.3389/fnins.2015.00221

Yu, L., Jin, W., Wang, J. X., Zhang, X., Chen, M. M., Zhu, Z. H., ... Zhang, Y. P. (2010). Characterization of TRPC2, an essential genetic component of VNS chemoreception, provides insights into the evolution of pheromonal olfaction in secondary-adapted marine mammals. Molecular Biology and Evolution, 27, 1467-1477.
Zapilko, V., \& Korsching, S. I. (2016). Tetrapod V1R-like ora genes in an early-diverging ray-finned fish species: The canonical six ora gene repertoire of teleost fish resulted from gene loss in a larger ancestral repertoire. BMC Genomics, 17, 83. https://doi.org/10.1186/ s12864-016-2399-6

\section{SUPPORTING INFORMATION}

Additional supporting information may be found online in the Supporting Information section at the end of the article.

How to cite this article: Hecker N, Lächele U, Stuckas H, Giere P, Hiller M. Convergent vomeronasal system reduction in mammals coincides with convergent losses of calcium signalling and odorant-degrading genes. Mol Ecol.

2019;28:3656-3668. https://doi.org/10.1111/mec.15180 